\title{
Causes of binder damage in porous battery electrodes and strategies to prevent it.
}

\author{
J. M. Foster ${ }^{\mathrm{a}}$, X. Huang ${ }^{\mathrm{b}}$, M. Jiang ${ }^{\mathrm{b}}$, S. J. Chapman ${ }^{\mathrm{c}}$, B. Protas ${ }^{\mathrm{d}}$, G. \\ Richardson ${ }^{\mathrm{e}}$ \\ ${ }^{a}$ Department of Mathematics, University of Portsmouth, Portsmouth PO1 2UP, UK. \\ ${ }^{b}$ General Motors Global R\&D, Warren, Michigan, US (48093). \\ ${ }^{c}$ Mathematical Institute, University of Oxford, Oxford OX2 6GG, UK. \\ ${ }^{d}$ Department of Mathematics $\&$ Statistics, McMaster University, Hamilton, ON, Canada \\ (L8P 2H4). \\ ${ }^{e}$ School of Mathematics, University of Southampton, Southampton SO17 1BJ, UK.
}

\begin{abstract}
The mechanisms for binder delamination from electrode particles in porous lithium-ion electrodes are considered. The problem is analysed using a model that makes use of a multiscale continuum description of the battery electrode and specifically accounts for the viscoelastic properties of the binder [9]. This model predicts the evolution of the stress fields in the binder in response to: (i) binder swelling due to electrolyte absorption during cell assembly, and; (ii) shrinkage and growth of the electrode particles during cell cycling. The model predictions provide a cogent explanation for morphological damage seen in microscopy images of real cathodes. The effects of altering electrode particle shape, binder rheology and cycling rates on binder delamination are all investigated and used to make suggestions on how electrode lifetimes could be extended.
\end{abstract}

Keywords: Lithium-ion polymer batteries, degradation mechanisms, binder delamination, mathematical models, viscoelastic constitutive relations, silicon anodes.

\section{Introduction}

Rechargeable lithium-ion cells can be used to store and release energy via electrochemical reactions. These lithium-based devices already domi-

Email addresses: jamie.foster@port.ac.uk (J. M. Foster), bprotas@mcmaster.ca (B. Protas), g.richardson@soton.ac.uk (G. Richardson) 
nate the consumer electronics sector and are becoming increasingly adopted for use in electric vehicles because they offer a high energy density, reasonable rate capability and long cycle-life [2, 16, 22]. They comprise two electrodes, one of which is negative (the anode) whilst the other is positive (the cathode). Each of these electrodes contain particles of active material, a carbon-doped polymer binder and pore space which is filled with electrolyte during operation; see figure 1. A working electrochemical cell is formed by sandwiching an anode and a cathode between two current collectors and electronically (but not ionically) separating them using a separator; see figure 2. On discharge lithium ions deintercalate from the negative electrode material, move into the positive electrode (where they are thermodynamically more stable) and then intercalate in the cathodic electrode particles. This flow of positive charge is compensated by an electronic current which flows from the anodic electrode particles through the polymer binder to the current collector, around an external circuit and into the cathode via the binder. On charging this process occurs in reverse [26].

Whilst a good amount of modelling work has been carried out aiming to improve cell performance (mostly based on Newman's seminal studies $[6,7,10,11])$ much less modelling has been done to elucidate what can be done to extend cell life time. Many reasons for cell fatigue and eventual failure have been proposed but the vast majority remain poorly-understood. The most commonly cited degradation mechanisms include the formation of solid-electrolyte interface layers $[1,30]$, gas generation [32] and severing of the electronic transport pathways either by loss of contact between the cell housing and current collector, the current collector and binder or between the binder and the internal electrode particles [18, 19, 20, 28]. The loss of electronic connectivity has been attributed to swelling of the polymer binder when it is submerged in electrolyte on cell assembly and volumetric changes in the active materials as they are cycled [3, 5, 12, 27, 31, 34].

Despite graphite being the most common negative electrode material there is currently a strong impetus to develop silicon-based alternatives which theoretically offer around 10 times larger capacities than their traditional counterparts [23]. The drawback of these Si-based anodes is that the active materials exhibit extremely large volumetric changes on (de)lithiation. Thus, understanding what can be done to minimise the damage caused by cycling is likely to be increasingly important moving forward $[15,17,21]$.

The aim of this work is to develop insight into ageing mechanisms that lead to morphological damage in porous electrodes. Specifically, we focus on the mechanical processes that give rise to delamination of the porous binder 

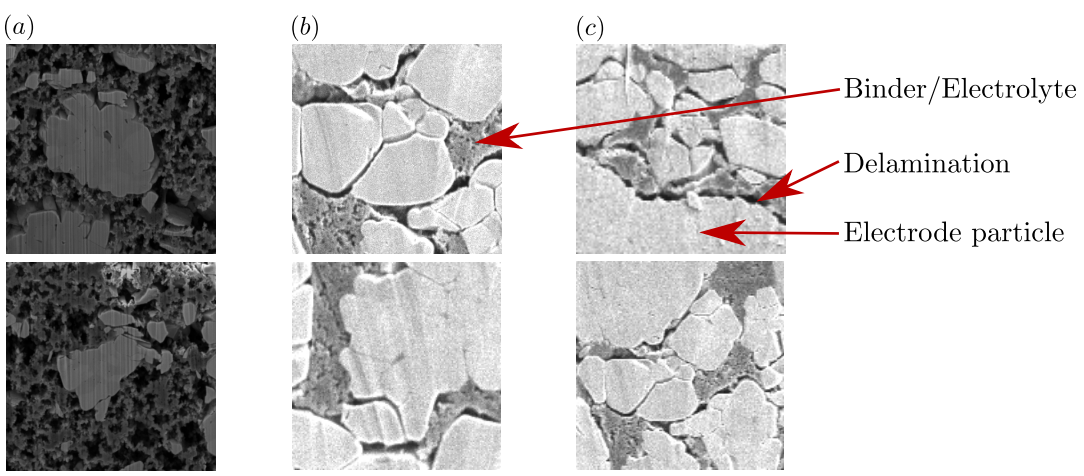

Figure 1: Focussed ion beam/scanning electron microscopy images of sections (approximately $10 \mu \mathrm{m} \times 10 \mu \mathrm{m}$ ) of NMC cathodes with a PVDF binder. The light grey and dark grey materials are the active material and binder respectively, whilst the black regions are pores space that accommodates the electrolyte when the cell is operational. The electrodes in column (a) were manufactured in G. R. Goward's group (McMaster Chemistry) whilst those in (b) and (c) are commercial devices. All images were harvested by G. A. Botton's group (McMaster Materials Science and Engineering) using the procedures described in [29]. The images in column (a) were taken before the electrode had been exposed to electrolyte. Those in column (b) were taken after the electrode had been immersed in electrolyte, constructed as part of a cell, undergone a single 'formation' cycle, removed from its housing and allowed to dry. The images in column (c) are of electrodes that have also formed part of a working device that has completed between 20 and 50 charge/discharge cycles before being dismantled for imaging.

material from the surface of the electrode particles, as illustrated in figure 1 , which leads to degradation in the cell performance (via capacity and lifetime loss) and also to decreased abuse tolerances. In an earlier study [9], we developed and analysed a multiscale mathematical model that predicts the mechanical response of porous electrodes to different actuation mechanisms occurring during the assembly and operation of the cell. The main objective of the current study is to show how different patterns of damage (i.e. forms of binder delamination), observed in real porous electrodes at different stages of assembly and use, can be explained in terms of the mechanical processes described by the model. A secondary objective is to use the model to explore how binder delamination is affected by the active particle shape, battery cycling rates and rheology of the polymer binder material. These results are used to formulate some general guidelines that can be used in the manufacture of potentially more robust porous electrode microstructures.

The structure of the paper is as follows: In the next section, we state the modelling assumptions, formulate the mathematical model and specify values of the material properties. We consider a reference case in which the 
electrode is constructed from circular (cylindrical) particles, embedded in a porous binder, and subject to standard assembly and cycling protocols. In $\S 3$, a comparison is made that shows good qualitative agreement between the predictions of the model and the morphological changes observed in FIB/SEM images of real electrodes. Following this, in $\S 4-6$, we study the effect of active particle shape, cycling rates and polymer rheology on binder delamination. Our concluding observations, including comments on how the reported findings could inform the design of more damage-resistant electrode morphologies, are made in $\S 7$.

\section{Modelling}

In a previous study [9], we developed a mechanical model describing the evolution of the stresses within a porous electrode based upon the following set of assumptions: (i) the electrode is two-dimensional (2D) and consists of cylindrical electrode particles (with circular cross-section) embedded in a porous viscoelastic binder material where the pores are filled with electrolyte (see Figures 1 and 2); (ii) the particles change volume in response to lithiation and delithiation (as the cell is charged and discharged); (iii) during manufacture, when the binder material initially comes into contact with the electrolyte, this fluid is partially absorbed causing swelling.

The full model uses a poroviscoelastic description that couples Darcy's law for the flow of the (liquid) electrolyte through the pores to a viscoelastic model (the standard linear model, or SLM) for the deformation of the binder material. Stress and deformation in the binder arise either as a result of binder material swelling on electrolyte absorption, or as a result of the growth/shrinkage of electrode particles embedded within the binder matrix as lithium is intercalated/deintercalated into their structure. Since these electrode particles have much greater mechanical moduli than the binder material, they are treated as rigid growing bodies (inclusions within the poroviscoelastic binder/electrolyte, see Figure 2). It was shown in [9] that, for a typical electrode, the permeability of the porous binder matrix is sufficiently large that, on the time scale of interest (i.e. that of battery charge and discharge), the electrolyte flows sufficiently freely through the binder so that, to a good approximation, the pressure within the electrolyte is uniform throughout the electrode. This leads to a significant simplification of the model in which the stresses and strains within the binder material decouple from the electrolyte flow. Under this simplification, the equations describing the evolution of the stress and strain in the binder phase reduce to a system of equations that are identical in form to a purely viscoelastic model. This 
simplified system of equations is outlined below (the full model can be found in $[9])$.

First, we have statements of a balance of forces and a definition of the (infinitesimal) strain tensor $\epsilon_{i j}$

$$
\frac{\partial \sigma_{i j}}{\partial x_{j}}=0, \quad(1) \quad \epsilon_{i j}=\frac{1}{2}\left(\frac{\partial u_{i}}{\partial x_{j}}+\frac{\partial u_{j}}{\partial x_{i}}\right)
$$

formulated in terms of the stress tensor $\sigma_{i j}$, the displacement vector $\boldsymbol{u}$ and the position vector $\boldsymbol{x}$. Here the indices take values $i, j=1,2$ and throughout we use the Einstein summation convention for repeated indices. Next, we decompose the stress $\left(\sigma_{i j}\right)$ and strain $\left(\epsilon_{i j}\right)$ tensors into their volumetric $(S$ and $E$ respectively) and deviatoric parts $\left(s_{i j}\right.$ and $\left.e_{i j}\right)$

$$
\begin{aligned}
& S=\frac{1}{2} \sigma_{k k}, \\
& E=\frac{1}{2} \epsilon_{k k},
\end{aligned}
$$

$$
\begin{aligned}
& s_{i j}=\sigma_{i j}-\delta_{i j} S, \\
& e_{i j}=\epsilon_{i j}-\delta_{i j} E .
\end{aligned}
$$

Finally, the governing equations are completed by a pair of constitutive equations (here we use the SLM) that relate the evolutions of the deviatoric and volumetric parts of the stress and strain

$$
\begin{gathered}
G_{\tau} \frac{\partial s_{i j}}{\partial t}+s_{i j}=G_{2} G_{\tau} \frac{\partial e_{i j}}{\partial t}+G_{1} e_{i j} \\
K_{\tau} \frac{\partial S}{\partial t}+S=K_{2} K_{\tau} \frac{\partial}{\partial t}\left(E-\beta_{a b s}(t)\right)+K_{1}\left(E-\beta_{a b s}(t)\right) .
\end{gathered}
$$

Here $K_{n}$ and $G_{n}$ for $n=1,2, \tau$ are material constants associated with the volumetric and deviatoric deformations, respectively. Material parameters with a numerical subscript are moduli (with units of pressure) while those with subscript $\tau$ denote a time scale. Finally, the function $\beta_{a b s}(t)$ describes the expansion of the polymer binder (as it absorbs the electrolyte) and is defined so that the long-term unstressed state has volumetric strain $E=$ $\beta_{a b s}(t)$.

In order to model the behaviour of a coin-cell electrode (albeit a twodimensional slab cell rather than a circularly cylindrical cell), we consider the problem described by equations (1)-(8) on a short-wide rectangle (of width $\sim 1 \mathrm{~cm}$ and height $\sim 100 \mu \mathrm{m}$ ) in which a uniform array of circular electrode particles (of radius $\sim 1-10 \mu \mathrm{m}$ ) are embedded; see figure 2 . On the lower surface of this rectangular electrode where it is affixed to the current 


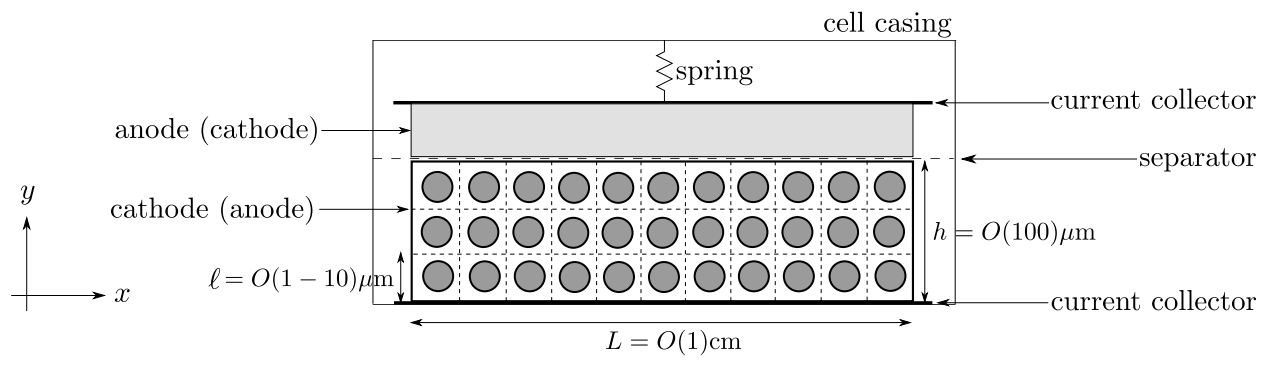

Figure 2: Schematic of the model geometry within a coin cell casing. The representative 'unit cell' of the microstructure is a square (with sides depicted by dashed lines) containing an electode particle (dark grey) within the cathode (anode) whilst the opposing electrode is shown in light grey.

collector we impose zero displacement. On the upper surface (where the electrode contacts the separator) we impose zero normal and zero tangential stress conditions accounting for the fact that the spring in a coin cell is only capable of applying a very weak compressive force (relative to what would be required to cause appreciable deformation of the electrode) on the electrode and that the separator materials are highly deformable (again relative to the electrode) [35].

(a)

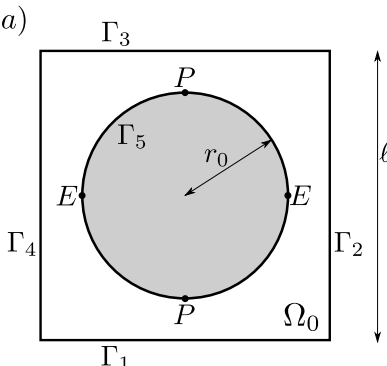

(b)

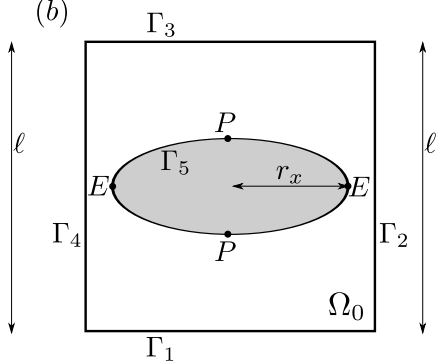

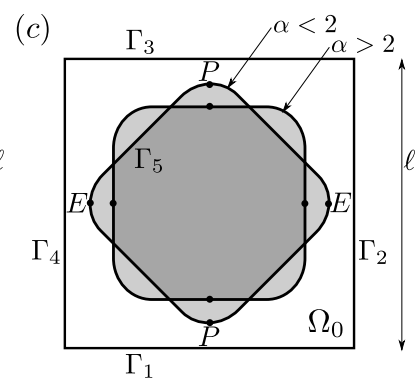

Figure 3: The three model microstructure (or unit cell) geometries, each of which is contained within a square with side lengths $\ell$. In all cases the boundary segments $\Gamma_{i}$ for $i=$ $1, \ldots, 4$ denote the lower, right-hand, upper and left-hand boundaries of the unit cell whilst $\Gamma_{5}$ denotes the interface between the electrode particle and surrounding binder/electrolyte porous medium. We also introduce the notation $P$ and $E$ (for 'pole' and 'equator') to denote upper-most and right-most positions on the particle surfaces - by the symmetry of the problem stresses $\left(\sigma_{P}\right.$ and $\sigma_{E}$ respectively) and strains at these positions are identical to those at the lower-most and left-most positions on the particle surfaces, respectively. Panel (a) depicts the geometry for the circular electrode particle of radius $r_{0}$; panel (b) shows the ellipsoidal electrode particle with major axis length $r_{x}$, and; panel (c) shows the geometry for a symmetric superegg with exponent $\alpha$ - this final shape is discussed in more detail in $\S 4$. 
The complexity of the geometry, consisting of a long thin polymer binder slab into which very large numbers of growing and shrinking microscopic electrode particles are embedded, renders direct solution of (1)-(8) intractable. This motivated us, in [9], to apply an upscaling procedure (also referred to as 'homogenization') that yields effective medium equations for the 'average' deformations on macroscopic lengthscales. Analysis of these effective medium equations allowed us to infer that the macroscopic deformation of the electrode occurs (to a very good approximation) almost entirely in the vertical direction ( $y$-direction), except in regions lying very close to the left- and right-hand edges of the electrode. An example macroscopic deformation is shown in the SI and full details are given in [9]. Consequently, the microscopic deformations around each electrode particle (away from the left- and right-hand edges) are almost identical. The problem of determining the microscopic stresses and deformations around the electrode particles can thus be reduced to the solution of the model inside a representative periodic box $\Omega_{0}$ around a single electrode particle (see figure 3). In order that the solution to this microscopic problem be consistent with the one-dimensional solution to the upscaled macroscopic problem, the boundary conditions on the microscopic problem (i.e. on the edges of the periodic box $\Omega_{0}$ ) must satisfy zero shear stress on all the exterior edges of the box, zero horizontal displacement on the vertical edges, and a non-zero (and a priori unknown) vertical displacement $\pm \lambda(t)$ on the horizontal edges:

$$
\begin{array}{lrrr}
\text { On } \Gamma_{1}: & u_{2}=-\lambda(t), & (9) & \sigma_{12}=0, \\
\text { On } \Gamma_{2}: & u_{1}=0, & (11) & \sigma_{12}=0, \\
\text { On } \Gamma_{3}: & u_{2}=\lambda(t), & (13) & \sigma_{12}=0, \\
\text { On } \Gamma_{4}: & u_{1}=0, & (15) & \sigma_{12}=0 .
\end{array}
$$

It is important to note the contrast between the displacement boundary conditions on the horizontal $\left(\Gamma_{1}\right.$ and $\left.\Gamma_{3}\right)$ and vertical boundaries $\left(\Gamma_{2}\right.$ and $\left.\Gamma_{4}\right)$. On the former a non-zero normal displacement is allowed whereas on the latter no normal deformation is allowed. We emphasize that this is not a modelling assumption; instead, it is a direct consequence of the large size of an electrode's width compared to its thickness, and the boundary conditions stated here are valid everywhere except very close to an electrode's circumferential edges — see [9] for full details. This strict confinement of the microstructure in the horizontal direction is central to the asymmetry 
in the results discussed later.

Appropriate boundary conditions on the edge of the circular electrode particle (see figure 3(a)), representing a time-dependent, but uniform, expansion from an initial radius $r_{0}$ to radius $r_{0}\left(1+g_{p}(t)\right)$ at time $t$, are

$$
\text { On } \Gamma_{5}: \quad u_{1}=x g_{p}(t), \quad(17) \quad u_{2}=y g_{p}(t) .
$$

These conditions are also appropriate for an arbitrarily shaped particle that grows in self-similar fashion (maintaining its shape as it grows, see figure 3) and correspond to a growth in particle area by a factor $\left(1+g_{p}(t)\right)^{2}$. The model assumes 'infinitesimal displacements' (a standard approach in computational continuum mechanics [14]) which, although less accurate than a nonlinear finite displacement approach, is more easily implemented and, except for applications with very large deformations, usually yields good results. This assumption allows the problem to be solved on the original undeformed body (reference domain) and avoids the difficulty of imposing boundary conditions on moving boundaries.

Finally, the function $\lambda(t)$, which is unknown a priori and appears in (9) and (13), is determined from the condition that there is zero net microscopic load in the $y$-direction, namely

$$
\int_{\Gamma_{1}} \sigma_{22} d S=0
$$

This requirement is necessary for the microscopic solution to be consistent with the upscaled (macroscopic) solution for which the compressive loading in the $y$-direction is identically zero (i.e. $\sigma_{22}^{\text {macro }} \equiv 0[9]$ ).

Provided electrodes are made carefully the binder and electrode particles are well connected immediately after drying (fabrication); see figure 1 . Consequently, we assume that initially the particles and binder are in full contact and that all components of the stress and deformation fields are zero. Moreover, we assume that binder and the electrode particles adhere to each other (as either a result of binder additives or van der Waals forces) and that binder delamination only occurs in response to significant tensile stresses on the surface of the electrode particles. Thus, when solving system (1)-(8) with boundary conditions (9)-(18) and constraint (19), we are particularly interested in monitoring normal stresses on the edge of the representative electrode particle (boundary segment $\Gamma_{5}$ ). 


\subsection{Parameter values}

The viscoelastic model for the porous PVDF (polyvinylidene fluoride) binder is calibrated by reference to experimental results published in [4] and [33] for PVDF's relaxation modulus. This time-dependent function is typically determined as a function of time by applying an instantaneous unit strain and measuring the resulting stress as a function of time. Wu et al. [33] parametrise their results in terms of the time-dependent relaxation modulus $Y(t)$, defined such that $\sigma(t)=Y(t) \epsilon(t)$, where $\sigma$ is the stress and $\epsilon$ is the strain (in this case a Heaviside function in time). They find that their results can be well approximated by the following Prony series for $Y(t)$ :

$$
Y(t)=Y_{\infty}+\sum_{i=1}^{5} Y_{i} \exp \left(-t / \tau_{i}\right),
$$

where $Y_{\infty}=0.52 \mathrm{MPa}, Y_{1}=0.045 \mathrm{MPa}, Y_{2}=0.89 \mathrm{MPa}, Y_{3}=0.0025 \mathrm{MPa}$, $Y_{4}=0.088 \mathrm{MPa}, Y_{5}=0.000207 \mathrm{MPa}, \tau_{1}=50 \mathrm{~s}, \tau_{2}=350 \mathrm{~s}, \tau_{3}=750 \mathrm{~s}$, $\tau_{4}=1500 \mathrm{~s}$ and $\tau_{5}=4000 \mathrm{~s}$. In order to calibrate the standard linear model (SLM) of viscoelasticity (which we use to describe the viscoelastic response of the binder) against this data we need to approximate this Prony series by a two term Prony series. We do this by noting that the dominant terms in (20) are those with coefficients $Y_{\infty}$ and $Y_{2}$ and so we lose little accuracy in approximating (20) by

$$
Y(t) \approx Y_{\infty}+Y_{2} \exp \left(-t / \tau_{2}\right)
$$

Notably, the experiments used to determine the relaxation modulus do not distinguish between the volumetric and deviatoric parts of the deformation. We therefore assume, as in [9], that the relaxation modulus is identical for volumetric and deviatoric deformations. Recalling that (21) is the stress resulting from a unit step increase of strain (described by the Heaviside function), we can write the solution for the stress associated with a general strain history $\epsilon(t)$ as the integral equation

$$
\sigma(t)=\left.\epsilon\right|_{t=0} Y(t)+\int_{0}^{t} Y\left(t-t^{\prime}\right) \dot{\epsilon}\left(t^{\prime}\right) d t^{\prime}
$$

By taking the Laplace transform of this equation and rearranging the result, it can be seen that this is equivalent to the differential equations (7)-(8) in which the coefficients have been set to

$$
K_{\tau}=G_{\tau}=350 \mathrm{~s}, \quad K_{2}=G_{2}=1.41 \mathrm{MPa}, \quad K_{1}=G_{1}=0.52 \mathrm{MPa} .
$$


When examining the effects of binder swelling we assume a temporal dependence of the electrolyte absorption given by

$$
\beta_{a b s}(t)=0.5 \tanh (t / \tau) \quad \text { choosing } \quad \tau=10 \mathrm{hrs},
$$

in equation (8). Since we are mainly interested in contrasting damage caused by swelling to that caused by cycling we simultaneously set the growth of the electrode particles to zero by writing $g_{p}(t) \equiv 0$ in $(17)-(18)$. This choice of $\beta_{a b s}$ means that for large times the zero stress state corresponds to the binders volume being increased by $50 \%$ which is in line with the observed PVDF swelling occurring in the cell construction process [3, 21, 27]. The constant $\tau$ characterises the time scale over which the binder absorbs electrolyte. Consistent with data for PVDF [3, 21, 27], we select $\tau=10 \mathrm{hrs}$, and note that some other binders, such as carboxymethylcellulose-based materials, can take much longer (several days) to fully swell in an electrolyte.

We base our simulations on a cathode containing nickel manganese cobalt oxide (commonly known as NMC) material. The active phase exhibits a volumetric expansion of around $4 \%$, and so when examining the effects of cell cycling we set

$$
g_{p}=0.02(1-\cos (t / \tau)) \ell \quad \text { choosing } \quad \tau=10 \mathrm{hrs},
$$

in (17)-(18) whilst setting $\beta_{a b s} \equiv 0$ in (8). The length $\ell$ that appears in (24) is the the length of the straight edges of the microstructures boundaries (see figure 3 ); throughout we take $\ell=5 \mu \mathrm{m}$. To ensure a fair comparison between the different electrode particle shapes (see $\S 4$ ), we fix the volume fraction of electrode material within the representative section of the microstructure as $\phi_{s}=0.4$. We note that this value of $\phi_{s}$ is significantly smaller than in many commercial devices. However, this choice allows us to make significant alterations in the particle shape while maintaining the size and periodicity of the microstructure thereby allowing a fair comparison of different particle geometries. The aim of our analysis is not to make quantitative predictions for specific cell compositions. In summary we take

$$
\ell=5 \mu \mathrm{m}, \quad \phi_{s}=0.4 \text {. }
$$

\section{Deformation around an array of circular electrode particles}

In this section stress and deformation fields are determined in the microstructure shown in figure $3(\mathrm{a})$, with $r_{0} \approx 0.7 \ell$ so that $\phi_{s}=0.4$, arising from (I) binder swelling (electrolyte absorption by the binder) and (II) 
volume changes to the electrode particles (lithium (de-)intercalation in the particles). Comparison is made between the model results and experimental imaging data. The numerical procedure used to solve the model is described in [9].

\subsection{Binder swelling}

As described in $\S 2$, the effects of binder swelling are investigated in an electrode, parametrised by (22) and (25), in which the binder swelling function is given by (23) and in which the electrode particles remain of constant size $g_{p}(t) \equiv 0$. The deformation and stress fields after the binder has absorbed the electrolyte are shown in figures 4(a) and 5, whilst figure 4(c) shows the evolution of the normal stresses, $\sigma_{P}$ and $\sigma_{E}$, at the points $P$ and $E$ (the pole and equator of the particle as illustrated in figure 3 ).

The plots in 4(c) show that after swelling, the normal stress at the point $P$ (top/bottom of the electrode particle) is tensile (positive), whereas at the point $E$ (right-hand/left-hand side of the particle) it is compressive (negative). This indicates that deformations induced by electrolyte absorption are likely to cause preferential delamination of the polymer binder from the top (and bottom) surfaces of the electrode particles, i.e. in planes parallel to the current collector and separator. This result can be understood intuitively by considering a growing solid medium confined within two rigid vertical walls. On swelling it has no choice but to decrease its internal stresses by flowing/deforming in the vertical direction (because of the horizontal confinement) - see figure 4(a). If the material also contains circular rigid inclusions (e.g., an electrode particle), this observation is still true; the growing material flows primarily in the vertical direction leading to tensile stress which could cause the material to pull away from the top and bottom surfaces of the circular inclusions. We reiterate that the firmer confinement in the horizontal direction is not assumed, rather, it is a consequence of the contrast between the thickness and lateral extent of electrodes.

\subsection{Volume changes of electrode particles in the cathode}

Here we examine the stress and deformation fields induced by the volume changes of electrode particles arising from electrochemical cycling in a positive electrode. Cathodes are constructed from electrode particles in a fully lithiated state (i.e. they are initially at their maximum volume). This contrasts with anodes which are constructed from electrode particles in a fully delithiated state. The mechanical effects of volume changes to the electrode particles are investigated in an electrode, parametrised by (22) and 

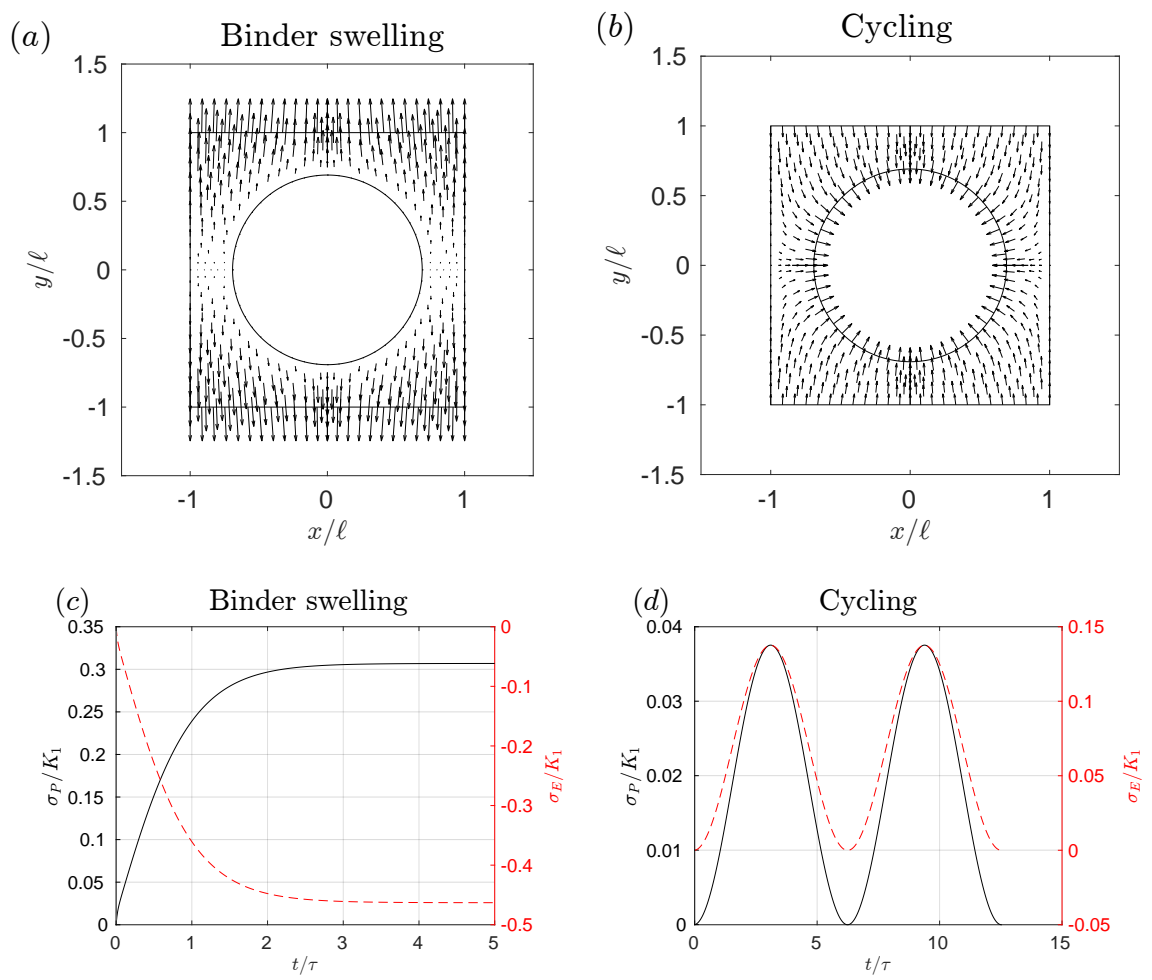

Figure 4: Panels (a) and (b) show the deformation fields generated by polymer swelling and cycling within a cathode respectively. In (a) the deformation field is shown after the binder has fully swollen (using the parameters discussed in §3.1) whereas in (b) the solution is shown at the peak of discharge, i.e., when the cathode particle is at its smallest (using the parameters discussed in §3.2). The arrow lengths in (a) are actual size whereas those in panel (b) have been magnified by a factor of 5 so that they are more readily visible. Panels (c) and (d) show the evolution of the normal stress at the points $P$ and $E, \sigma_{P}$ (black solid curves) and $\sigma_{E}$ (red dashed curves), on the particle surface when deformations are forced by: (a) polymer swelling due to electrolyte absorption, and; (b) volumetric changes of the cathodic active material due to cycling. In panel (a) the simulation parameters are given in $\S 3.1$; the parameters for panel (b) are those in $\S 3.2$. 

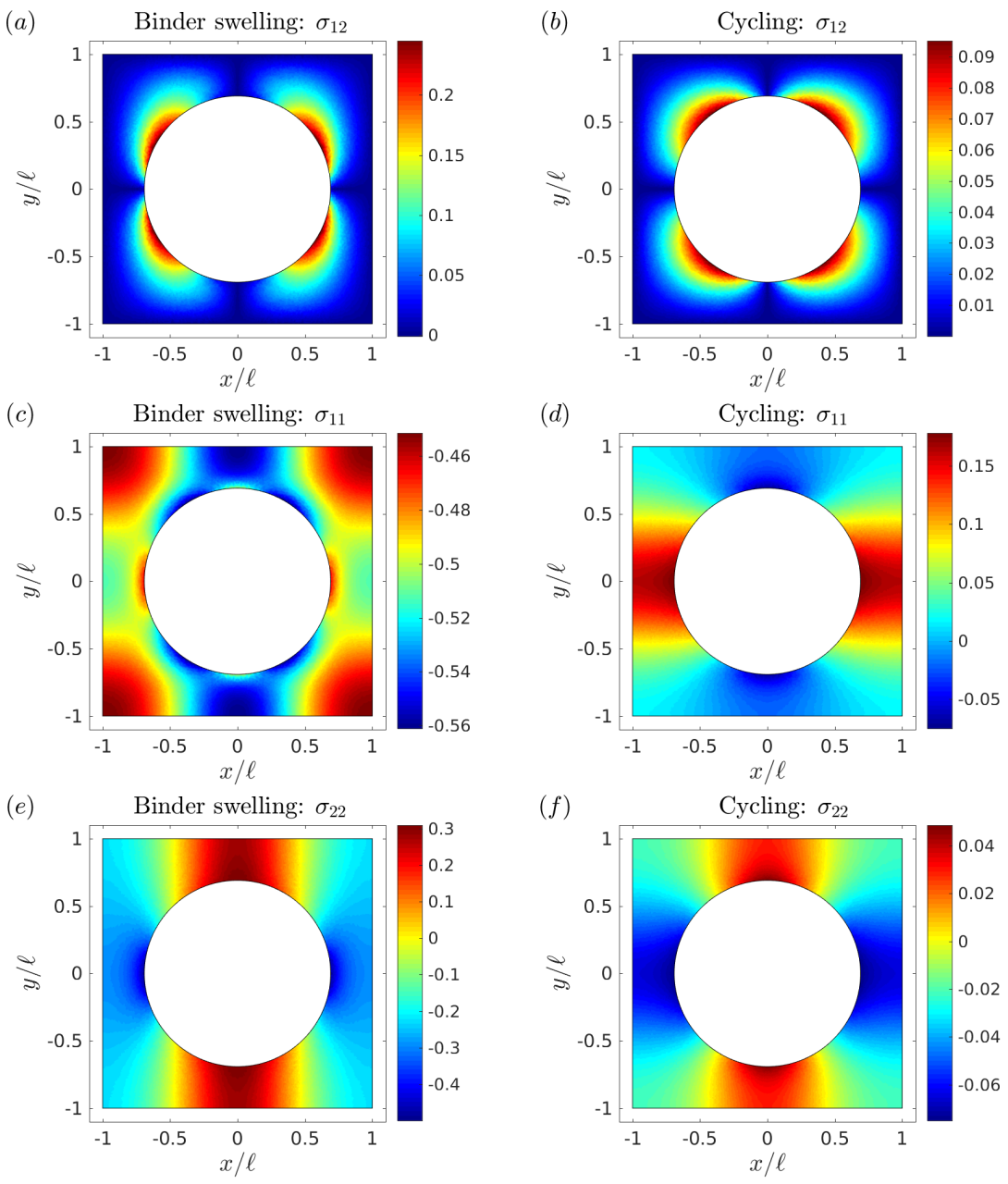

Figure 5: The left-hand column shows plots of the different components of the stress tensor after the binder has fully swollen $(t=50 \mathrm{hrs})$. The simulation parameters are those discussed in $\S 3.1$. Note that $\sigma_{P}>0$ corresponding to the binder being in tension whereas $\sigma_{E}<0$ corresponding to the binder being in compression. The right-hand column shows plots of the different components of the stress tensor in the cathode at the peak of the first discharge $(t \approx 3.1 \mathrm{hrs})$. The simulation parameters are those discussed in $\S 3.2$. Note that both $\sigma_{P}, \sigma_{E}>0$ corresponding to the binder being in tension. In all panels the stresses are measured in units of $K_{1}=0.52 \mathrm{MPa}$.

(25), in which the binder does not swell $\beta_{a b s} \equiv 0$ and in which electrode particle expansion is described by (24).

The components of the deformation and stress field at the peak of the first discharge (corresponding to $t \approx 3 \mathrm{hrs}$ ) are shown in figures $4(\mathrm{~b})$ and 5 
respectively. The evolution of the normal stresses at the points $P$ and $E$, i.e. $\sigma_{P}$ and $\sigma_{E}$, on the particle surface are shown in figure 4(d) for two complete cycles. In contrast to the deformations driven by electrolyte absorption, these electrode deformations induced by the growth and shrinking of particles cause tension in the binder at all positions on the particle surface. Furthermore, the normal stress (directed outward) at the right-hand (and left-hand) sides of the electrode particle are larger than those at the top (and bottom), indicating that volumetric changes in the electrode particles are likely to drive delamination of the binder at the left and right edges of the electrode particle more strongly. The reasons for this are straightforward to understand; throughout the cycle the particles are smaller than their original size and therefore the surrounding polymer material is in tension. The size of this tension is larger at the left- and right-hand sides because the adjacent boundaries of the unit cell are fixed, whereas the top (and bottom) of the unit cell can deform thereby reducing the tension.

\subsection{Agreement with experimental studies}

The main conclusions regarding the contrast between the deformations induced by polymer swelling and cycling are in good qualitative agreement with experimental studies. As discussed in $\S 2$, and in agreement with the initial conditions in our model, FIB/SEM images of research-grade NMC cathodes that have neither been immersed in electrolyte, nor undergone cycling, exhibit very little delamination, see figure 1 and also the cathode images presented in [13]. However, electrodes that have been immersed in electrolyte and have undergone just a single formation cycle do exhibit delamination - as shown in the images in figure 1 (details on the electrode composition and preparation are given in $[8,29]$ ). Moreover, this delamination occurs preferentially in planes parallel to the current collector and separator. Since a single formation cycle is unlikely to cause significant structural damage, the delamination seen in these devices can be largely attributed to swelling of the binder as it absorbs electrolyte. Our mathematical model thus gives a coherent explanation for this damage, in particular, showing that binder swelling gives rise to large tensile stresses on the top and bottom surfaces of the electrode particles (which run parallel to the current collector and separator), to which it seems likely that delamination can be attributed.

In-depth comparisons of both NMC and high-energy NMC (HE-NMC) electrodes that have been both immersed and (a) undergone a single formation cycle, and (b) undergone longer term cycling (20-50 full cycles), have been carried out in $[8,29]$. These studies demonstrate that those electrodes 
that have been more heavily cycled show increased signs of structural damage. For example, in [8] it was demonstrated that the components of the effective electronic conductivity tensor decreased by $\sim 4 \%$ for NMC and $\sim 6 \%$ for HE-NMC electrodes, respectively. Furthermore, this decrease in effective conductivity was accompanied by a decrease in the amount of contact between the binder and active material surfaces of $\sim 9 \%$ for $\mathrm{NMC}$ and $\sim 12 \%$ for HE-NMC electrodes, suggesting that the overall drop in conductivity can, at least partially, be attributed to further delamination driven by cycling. The results for both the effective conductivity tensor and relative surface contact area were given for each of the three directions within the electrode and no discernible trend for increased/decreased damage in a particular direction was observed. A similar image analysis was carried out in [29] and it was shown there that the amount of contact between the binder and active material decreases roughly uniformly through the depth of the electrode, see figures 4 and 7 in [29]. Once again, the predictions of our mathematical model are in line with the experimental observations; they show that electrochemical cycling of the cathode gives rise to tensile stresses on the surface of the electrode particles that could lead to delamination of the binder from these areas (however, it is notable that these stresses are considerably smaller than the stresses arising from the more dramatic volume changes occurring as a result of electrolyte absorption by the binder). Moreover, the predictions of our mathematical model are also consistent with uniform damage observed throughout the electrode depth. We attribute the rather small changes in effective conductivity and surface contact area to the relatively small volume changes of the NMC material $(\sim 2-4 \%)$ and speculate that these effects are likely to be considerably more drastic for either metal flourides or metal chlorides compound cathode materials (which can change their volumes on (dis-)charge by up to $\sim 30 \%$ ) or for silicon-based anode materials (which have volumetric changes of up to $\sim 400 \%$ ) [25].

\section{The impact of the shape of electrode particles on delamination}

In this section we investigate the effects of electrode particle shape on the delamination process by calculating the stress and deformation fields for two families of smoothly varying particle shapes, namely, (i) fixed-area ellipses and (ii) fixed-area 'supereggs'. In case (i) we consider constantarea ellipses with volume fractions $\phi_{s}=0.4$ (i.e. they occupy $40 \%$ of the surrounding periodic box) and adjust the major $(x-)$ axis, $r_{x}$, in order to probe the effects of aspect ratio on stress build up. In case (ii) the superegg 
shape is described by the relation $\left(x / r_{e}\right)^{\alpha}+\left(y / r_{e}\right)^{\alpha}=1$ for $r_{e}>0$ and $1<\alpha<\infty$. A sketch of this family is given in figure 3(c) where we adjust the values of the parameter $\alpha$. For each different value of $\alpha$ we fix a different value of $r_{e}$ so that the each superegg also has a volume fraction $\phi_{s}=0.4$. This procedure allows us to explore the effect of corners in the particle shape on delamination.

\subsection{Binder swelling}

We initially explore the effects of changing particle shape on binder swelling-induced damage. In all cases the effects of binder swelling are investigated in an electrode, parametrised by (22) and (25), in which the binder swelling function is given by (23) and in which the electrode particles remain of constant size $g_{p}(t) \equiv 0$. In figure 6 we find, as was also the case for circular particles, that both for (i) elliptic particles with $0.5 \ell \leq r_{x} \leq 0.95 \ell$ (which is more or less the entirety of the allowable range of $r_{x}$ while maintaining $\phi_{s}=0.4$ ) and for (ii) superegg-shaped particles with $1.1 \leq \alpha \leq 8$, the normal stress at $P$ is always positive (binder in tension) whereas that at $E$ is always negative (binder in compression). Thus, even for ellipses and supereggs, delamination driven by binder swelling is likely to occur along planes parallel to the current collector (i.e. on the top and bottom surfaces of the electrode particles). We also find that the maximum value of the stress at $P, \sigma_{P}$, is a decreasing function of both $r_{x}$ and $\alpha$, see figure 7(a) and (c), which suggests that having a relatively large portion of the particle surface running parallel to the current collector acts to reduce the tensile stress giving rise to delamination. However, this effect is offset by the greater extent of the surface that is under tension when either $r_{x}$ or $\alpha$ are large (and therefore the larger area that is liable to delamination) see figure 6 . In other words, shapes with high curvature on their top and bottom surfaces $\left(r_{x}\right.$ and $\alpha$ small) tend to concentrate stress there whereas shapes with flat top and bottom surfaces ( $r_{x}$ and $\alpha$ large) tend to dissipate the stress more evenly across these surfaces. Which arrangement is better at limiting the damage caused by swelling of the polymer is an open question and will depend to a large extent on the critical stress required to initiate delamination. In this context we point to the many images in $[8,13,29]$ and to those contained in figure 1 which seem to show a tendency for larger delamination zones on the flatter surfaces. Finally we note that $\sigma_{E}$ varies little as these parameters change. 

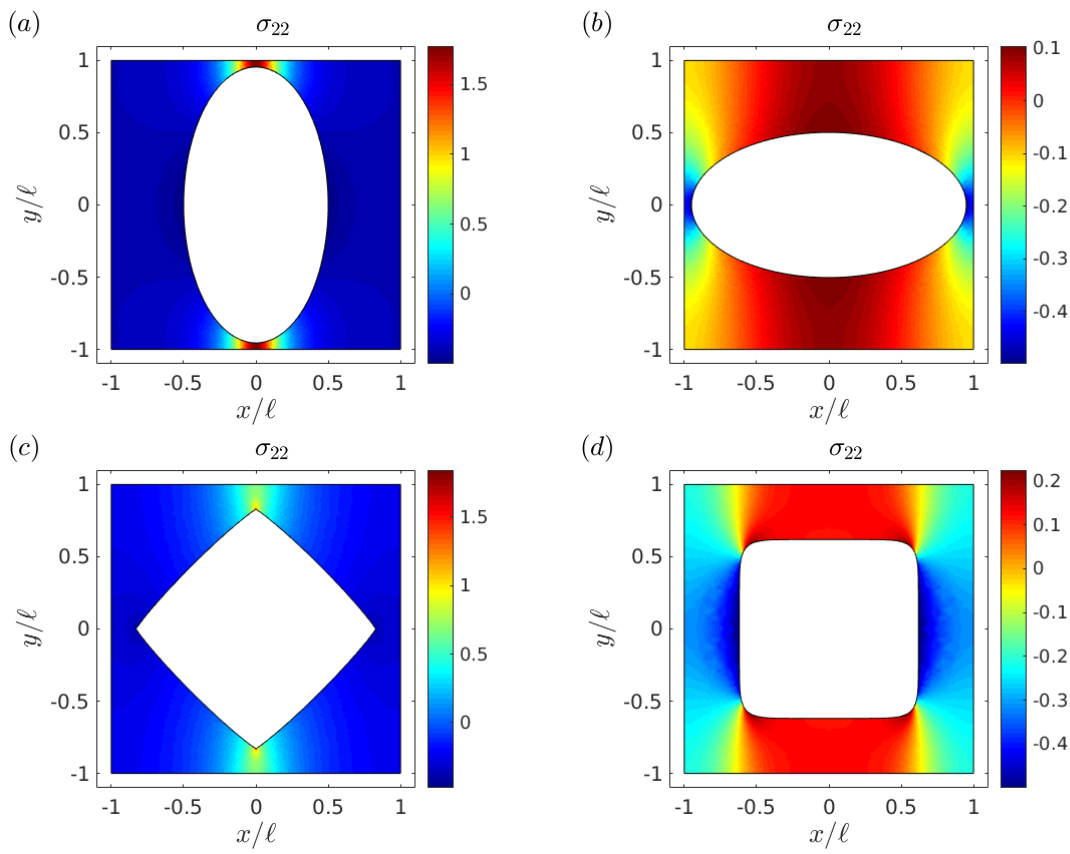

Figure 6: Plots of $\sigma_{22}$ in the cathode after the binder has absorbed electrolyte and swollen. The simulation parameters are those discussed in $\S 4.1$. The stresses are measured in units of $K_{1}=0.52 \mathrm{MPa}$. Panels (a)-(d) show the field for a prolate ellipse $\left(r_{x}=0.5 \ell\right)$, oblate ellipse $\left(r_{x}=0.95 \ell\right)$, diamond-shaped superegg $(\alpha=1.1)$ and a square-shaped superegg $(\alpha=8)$.

\subsection{Volume changes of electrode particles}

Once again mechanical effects of volume changes to the electrode particles are investigated in an electrode, parametrised by (22) and (25), in which the binder does not swell $\beta_{a b s} \equiv 0$ and in which electrode particle expansion is described by (24). As in the circular case we find that cycling of cathode particles leads to positive normal stresses (i.e. to tension) everywhere along the electrode particle surfaces. This arises because the electrode is manufactured from an unstressed binder and fully lithiated cathode particles in their largest-volume state so that subsequent cycling leads to shrinkage of the electrode particles and induces tension in the binder. For circular particles (see §3) we found that cycling leads to maximal tensile stresses at the point $E$, a trend that also appears to hold for the majority of ellipsoidal particles that we considered (the exception being those with values of $r_{x}$ close to its minimum possible value $r_{x}=0.5 \ell$ ). In this exceptional case the particles are so tall and thin that there is little binder material between an electrode 

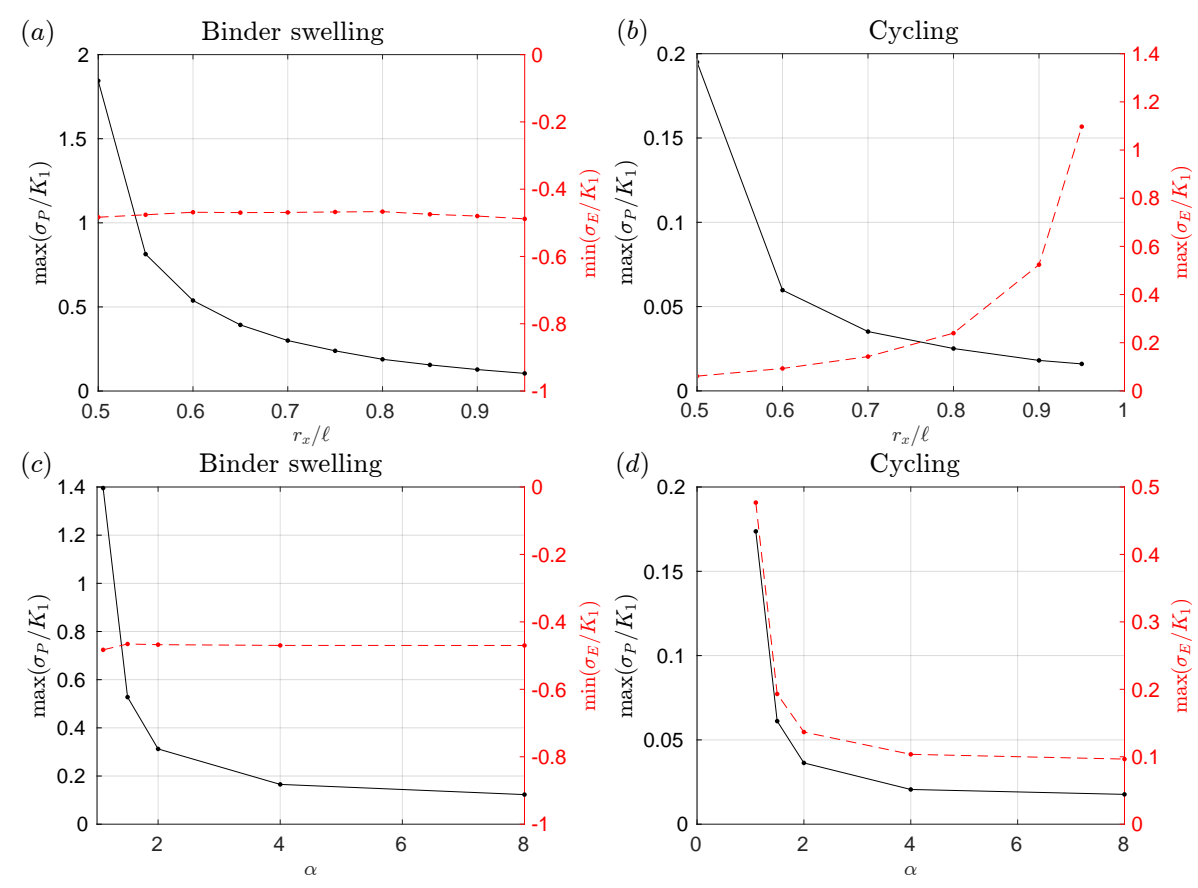

Figure 7: Variations of the maximum value (w.r.t. time) of $\sigma_{P} / K_{1}$ (black solid curves) and the minimum value of $\sigma_{E} / K_{1}$ (red dashed curves) as a function of $r_{x} / \ell$ (panels (a) and (b)) and $\alpha$ (panel (c) and (d)). Panels (a) and (c) are for when the problem is forced by swelling of the binder, i.e., when simulations are carried out using the parameters (22) and (25) whilst $g_{p}(t) \equiv 0$ and the binder swelling function is taken to be (23). Panels (b) and (d) are for a cathode undergoing cycling, i.e., when simulation are carried out using the parameters (22) and (25) whilst taking $\beta_{a b s}(t) \equiv 0$ and the electrode particle expansion is described by (24).

particle and its neighbours immediately above and below it. Therefore, even though the height of the unit cell changes to dissipate the net tension across the unit cell, this is not enough to significantly reduce the tension in the small region of binder separating vertically neighbouring electrode particles. Correspondingly, for small $r_{x}$, there is also a relatively large thickness of binder between neighbouring particles in the horizontal direction, which forms a cushion acting to absorb horizontal tension stresses. Thus, by altering the aspect ratio of the electrode particles (assuming ellipses), one may trade normal stress at $P$ for normal stress at $E$ (see figure $7(\mathrm{~b})$ ). Carrying out the same parameter sweep reveals that the normal stresses at both $P$ and $E$ decrease with increasing $\alpha$ (see figure $7(\mathrm{~d})$ ). Thus, it appears that by making the electrode particles square, and aligning a straight edge of 
those squares with the current collector (and separator) one might be, in principle, able to mitigate the damage associated with cycling everywhere on the particle surface. Once again the caveat to this is that this type of configuration leads to a greater extent of surface subject to reasonably large tensile stresses, although the maximum tensile stress along such a surface is considerably lower than for the circular particle.

\section{The impact of cycling rate}

It is well-known that cycling at high rates accelerates degradation in lithium-ions cells [24]. In order to investigate this phenomenon using our model, we return to the geometry based on the circular electrode particles shown in figure 3(a) and consider first a cathode. The effect of altering cycling rate is achieved in the model by adjusting the value of $\tau$ in the function $g_{p}(t)$, see $(24)$, that appears in the boundary conditions (17)-(18). By varying the cycling time scale $\tau$ from $1000 \mathrm{hrs}$ to $0.01 \mathrm{hrs}$ in increments of factors of 10, the time taken to carry out a single cycle is altered from approximately $3100 \mathrm{hrs}$ to $0.031 \mathrm{hrs}$ respectively. We note that, although the slowest and fastest of these rates are unrealistic, they do serve as an informative limiting cases.

In figure 8(a) we show the value of $\sigma_{E}$, the normal stress at the point $E$ at the side of the particle, over the first two cycles for each of the six different rates. This plot shows a monotonic increase the maximum value of $\sigma_{E}$ with cycle rate, a result that is consistent with the experimental observation that degradation rates increase with increases in the charge/discharge rate. We also observe that for the slowest and fastest cycling rates the deformation is almost elastic, and that it is only at the intermediate cycling rates that viscoelastic relaxation is evident. This can be understood in terms of the constitutive properties as follows: at fast rates deformation is almost entirely elastic and governed by the (smaller) instantaneous modulus, whereas at slow rate creep occurs much more quickly than the volume changes of electrode particle and so deformation is dominated by the (larger) modulus associated with longer time scales.

In figure 8(b) we show analogous results for an anode. These simulations were carried out using identical parameters to those for the cathode with the exception that the particle swelling function is $g_{p}(t)=-0.02(1-\cos (t / \tau)) \ell$, i.e., it has the opposite sign with respect to the swelling function in the cathode. Similar trends are observed, but, for the majority of the evolution (and the majority of the different cycling rates) the value of $\sigma_{E}$ is now negative (binder in compression). This is to be expected, because in an anode 
the particles are manufactured in a delithiated state and so the zero-stress initial conditions are for a configuration in which the electrode particles are at their minimal volume (fully delithiated). As for the cathode, the slowest cycle rates result in an almost entirely elastic response of the binder. As the cycle rate increases, viscoelastic creep becomes observable until, at sufficiently fast cycling rates, an elastic limit is reached which is governed by the instantaneous modulus. At intermediate rates viscoelastic creep causes $\sigma_{E}$ to change sign and briefly become positive (i.e. the binder goes into tension) at the peak charge (when the electrode particles return their smallest size). Interestingly, it therefore seems that intermediate rates (for practical purposes these would usually be considered fast) are most damaging to the anode.

In summary, faster cycling rates upwards of around $1 C$ are more damaging for both cathodes and anodes, but for quite different reasons.
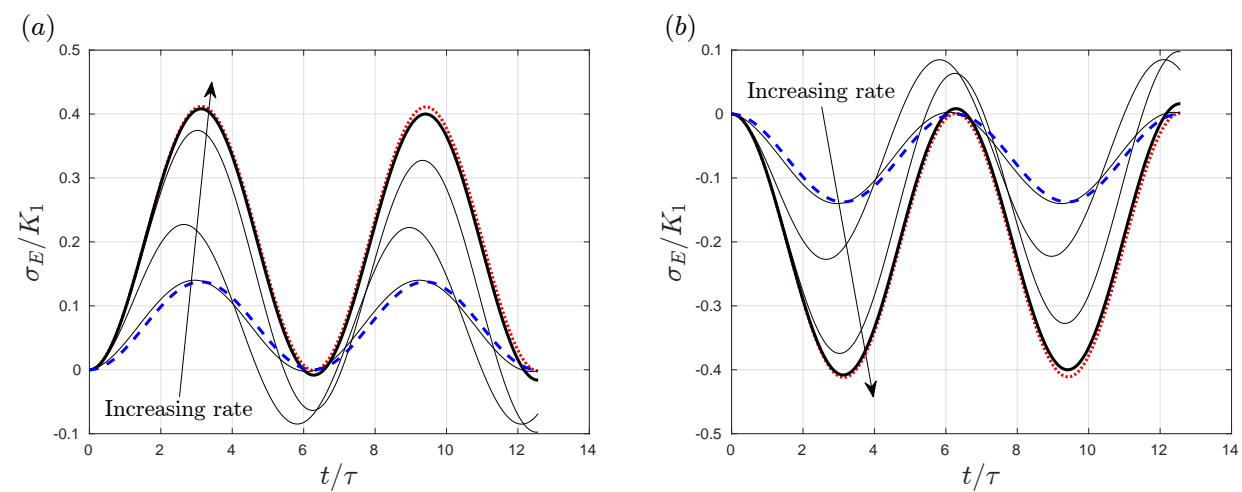

Figure 8: Panel (a) shows plots of the variation of $\sigma_{E} / K_{1}$ as a function of time for different cycling rates in a cathode and panel (b) shows the same quantity but for an anode. Here $K_{1}=0.52 \mathrm{MPa}$. In both cases the blue (dashed) curves corresponds to the slowest cycling rate (a full cycle takes $3100 \mathrm{hrs}$ ) whilst the red (dotted) curve shows the results for the fastest rate (a full cycle takes $0.03 \mathrm{hrs}$ ). The black curves take intermediate values between the two in increments of factors of 10 - see the discussion $\S 5$ for further details.

\section{The impact of polymer rheology}

The SLM of viscoelastic materials depends on six distinct mechanical moduli/relaxation timescales. Here the effects of altering these parameters are considered. 


\subsection{Binder swelling}

First we analyse the effect of rheological modifications on the stresses caused by polymer swelling. Simulations are parametrised by (22) and (25) unless otherwise stated, whilst $g_{p}(t) \equiv 0$ and the binder swelling function is given by (23). The variation of the stresses caused by altering the shear relaxation timescale $G_{\tau}$ is minimal. Altering the volumetric relaxation timescale $K_{\tau}$ gives a much larger variation with increases in the timescale resulting in a monotonic increase in the stresses $\sigma_{P}$, see the SI. The maximum value of $\sigma_{P}$ saturates for both small and large values of $K_{\tau}$ indicating that either the instantaneous (for large $K_{\tau}$ ), or long-term (for small $K_{\tau}$ ) elastic limit has been reached. For polymer swelling, altering the values of both short-term shear and bulk moduli, $G_{2}$ and $K_{2}$, respectively, has little effect on the maximal values of the stress. This can be understood readily, since binder swelling occurs on a time scale of around 10hrs that is much larger than the viscoelastic relaxation timescale; the deformation is thus almost entirely governed by the long-term moduli, $G_{1}$ and $K_{1}$. The most drastic changes in the maximal value of the stresses arises from changing these longterm moduli, in particular; (i) increasing $K_{1}$ and $G_{1}$ together keeping the ratio fixed, $\sigma_{P}$ increases linearly with $K_{1}$ and $G_{1}$ (ii) increasing $K_{1}$ while keeping $G_{1}$ fixed $\sigma_{P}$ increases with $K_{1}$ but sub-linearly, and (iii) increasing $G_{1}$ while keeping $K_{1}$ fixed $\sigma_{P}$ increases, but not so strongly as with $K_{1}$ (see the figures in the SI). We therefore conclude that the most effective strategy for reducing delamination due to binder swelling is to reduce the long-term moduli - particularly $K_{1}$, associated with the volumetric part of the deformation - and to ensure that the bulk relaxation timescale remains short enough that only these long-term moduli are relevant.

\subsection{Volume changes of electrode particles}

We now examine an analogous set of results for delamination induced by cycling. Simulations are parametrised by (22) and (25), unless otherwise stated in the text or indicated by axis labels, whilst $\beta_{a b s}(t) \equiv 0$ and the expansion of the electrode particles is described by (24). Here, increasing the relaxation time scale $G_{\tau}$ associated with the shear part results in an increase of $\sigma_{P}$, see the figures in the SI. On the other hand, increasing $K_{\tau}$ has only a small effect. It is interesting to note the direct contrast between this result and the analogous one in the binder-swelling case. The fact that $G_{\tau}$ is the more important time scale in the cycling case, whereas $K_{\tau}$ is more important for binder swelling, seems to be a reflection of the fact that in the binder swelling case the binder has to cope mainly with a change in size, whereas 
for cycling it is more a change in shape. For cycling-induced delamination, once again, we find that variations in the instantaneous moduli $\left(G_{2}\right.$ and $K_{2}$ ) have very little effect while the long-term moduli $\left(G_{1}\right.$ and $\left.K_{1}\right)$ have appreciable effects (see the figures in the supporting information). This can be explained on the same grounds as those discussed in the paragraph above. One interesting feature observed here is that an increase in $K_{1}$ results in a (beneficial) decrease of $\sigma_{P}$, yet a (detrimental) increase in $\sigma_{E}$.

\section{Conclusions}

We have used a mechanical model [9] to predict the stresses in the viscoelastic (polymer) binder material of commercial lithium ion electrodes induced either by: (i) polymer swelling caused by electrolyte absorption by the binder, or by (ii) cyclic growth/shrinkage of the electrode particles embedded within the binder as these particles lithiate and delithiate. The stresses thus calculated have been used to infer likely mechanisms for, and the sites of, binder delamination from the electrode particles. In particular, the model predicts that binder swelling on absorption of electrolyte gives rise to large tensile stresses on electrode particle surfaces that run parallel to the current collector and separator, from which we infer that binder-swelling induced delamination is likely to occur in these planes as, indeed, is observed in FIB/SEM images of newly constructed battery cathodes [13]. In contrast, cell cycling of the cathode (and the attendant volume changes of electrode particles), induces smaller tensile stresses all around the electrode particles, which are however more pronounced in planes perpendicular to the current collector. This suggests that electrode damage attributable to cell cycling is likely to result in delamination on all surfaces of electrode particles, but particularly so in planes perpendicular to the current collector and the separator. These observations are in good qualitative agreement with previous studies based on analysis of microscopy images [8, 29] and these findings also concur with the images shown here, see figure 1.

Owing to the microscopy images and degradation data available, we based our simulations on an NMC cathode with a PVDF binder. This active material and binder exhibit volumetric expansions of $\sim 4 \%$ and $\sim 50 \%$ on (de)lithition and immersion in electrolyte respectively. As a consequence the model predicts that the stresses due to binder swelling are around an order of magnitude larger than those due to cycling, see e.g. figures 4 and 5. This relation between stress magnitudes arising from the two different actuation mechanisms is likely to be different in cases where (i) the swelling of the active material (e.g. for graphite, metal flourides, metal chlorides or 
silicon-based materials) is more pronounced or (ii) where the binder swelling is less pronounced.

We explored the impact of electrode particle shape on the stress distribution within the binder finding that the maximum tensile stresses induced by binder swelling can be reduced by aligning the longer axis (i.e. the straighter edge) of the particles with the current collector, thereby distributing the largest stress (which occurs at the top and bottom of the particles) over a larger area. However this reduction in maximum stress comes at the expense of exposing a greater surface area to reasonably larger tensions, which therefore might potentially cause more extensive damage. We also explored the effects of altering particle shape on cycling-induced delamination. Once again, we found that by aligning the longer axis (i.e. the 'straighter edge') of the particles with the current collector that the maximum tensile stresses in the binder at the surface of the electrode particles can be reduced.

Next, we examined the impact of cycling rate. Here, we found that increasing cycling rate leads to conditions where increased amounts of binder delamination are likely to be seen in cathodes. Counter-intuitively, in anodes, we found that relatively fast cycling rates of around $1 \mathrm{C}$ were the most damaging. Further accelerating the cycling to very high rates of $0.1 \mathrm{hrs}$ or faster serves to decrease damage in the anode - see the discussion in $\S 5$ for an explanation of this phenomenon.

Finally, we examined the role of the polymer binder rheology. Here, we found that for the majority of practically relevant cycling rates, slower than around one full cycle per hour, the most important material properties are the long-term moduli. At increased cycling rates the relaxation time scales also play an important role. The most fruitful avenues for materials scientists to improve binders therefore seem to be to decrease both the relaxation time scales (to ensure that even for large cycling rates the deformations are predominantly elastic) and the long-term moduli (associated with both shear and bulk deformations).

\section{Acknowledgements}

Thanks to M. Z. Tessaro, K. J. Harris, H. Liu, C. Wiktor, G. R. Goward, G. A. Botton, I. Hallalay and C. P. Please for stimulating discussions regarding this work. The electron microscopy data included in this paper was acquired at the Canadian Centre for Electron Microscopy, a national facility supported by the Natural Sciences and Engineering Research Council of Canada (NSERC) and McMaster University. Funding for this research was 
provided by Automotive Partnership Canada (APC) and General Motors of Canada.

\section{References}

[1] D. Aurbach, E. Zinigrad, Y. Cohen \& H. Teller. Solid State Ion., 148 (2001) 405.

[2] P. G. Bruce, B. Scrosati \& J. M. Tarascon. Angew. Chem. Int. Ed., 47 2930 (2008).

[3] L. Chen, X. Xie, J. Xie, K. Wang \& J. Yang. Binder effect on cycling performance of silicon/carbon composite anodes for lithium ion batteries. J. Appl. Electrochem., 36(10):1099-1104 (2006).

[4] Z. Chen, L. Christensen \& J. R. Dahn. Mechanical and electrical properties of poly(vinylidene fluoride-tetrafluoroethylene-propylene)/super-s carbon black swelled in liquid solvent as an electrode binder for lithiumion batteries. J. Appl. Poly. Sci., 91(5):2958-2965 (2004).

[5] S. Chou, Y. Pan, J. Wang, H. Liu \& S. Dou. Small things make a big difference: binder effects on the performance of $\mathrm{Li}$ and $\mathrm{Na}$ batteries. Phys. Chem. Chem. Phys., 16:20347 (2014).

[6] M. Doyle and J. Newman. Analysis of capacity-rate for lithium batteries using simplified models of the discharge process. J. Appl. Electrochem., 27:846-856, 1996.

[7] M. Doyle and J. Newman. Comparison of modeling predictions with experimental data from plastic lithium ion cells. J. Electrochem. Soc., 143:1890-1903, 1996.

[8] J. M. Foster, A. Gully, H. Liu, S. Krachkovskiy, Y. Wu, S. Schougaard, M. Jiang, G. Goward, G. A. Botton \& B. Protas. A homogenization study of the effects of cycling on the electronic conductivity of commercial lithium-ion battery cathodes. J. Phys. Chem., 119(22):1219912208, (2015).

[9] J. M. Foster, S. J. Chapman, G. Richardson \& B. Protas. A mathematical model for mechanically-induced deterioration of the binder in lithium-ion electrodes. submitted for publication (see arXiv:1608.04847), (2016). 
[10] T. F. Fuller, M. Doyle, J. Newman. Relaxation phenomena in lithiumion insertion cells. J. Electrochem. Soc. 143:982-990, 1994.

[11] T. F. Fuller, M. Doyle and J. Newman. Simulation and optimisation of the dual lithium insertion cell. J. Electrochem. Soc., 141:1-10, 1994.

[12] A. Guerfi, M. Kaneko, M. Petitclerc, M. Mori \& K. Zaghib. J. Power Sources, 163 (2007) 1047.

[13] A. Gully, H. Liu, S. Srinivasan, A. K. Sethurajan, S. Schougaard \& B. Protas. Effective transport properties of porous electrochemical materials - a homogenization approach. J. Electrochem. Soc., 161(8):E3066-E3077. (2014).

[14] M. E. Gurtin, E. Fried, L. Anand. "The Mechanics and Thermodynamics of Continua", Cambridge University Press, (2010).

[15] Y. M. Kang, J. Y. Go, S. M. Lee \& W. U. Choi. Electrochem. Commun., 9:1276-1281 (2007).

[16] B. Kang, Y. S. Meng, J. Berger, C. P. Grey \& G. Ceder. Electrodes with high power and high capacity for rechargeable lithium batteries. Science, 311:977-980 (2006).

[17] I. S. Kim \& P. N. Kumta. J. Power Sources, 136:145-149 (2004).

[18] R. Kostecki \& F. McLamon. Electrochem. Solid-State Lett., 7 (2004) A38.

[19] G. Liu, H. Zheng, X. Song, \& V. S. Battaglia Particles and Polymer Binder Interaction: A Controlling Factor in Lithium-Ion Electrode Performance J. Electrochem. Soc., 159: A214-A221 (2012).

[20] I. Konalenko, B. Zdyrko, I. Koalenko, B. Hertzberg, R. Burtoyy, C. F. Huebner, T. F. Fuller, I. Luzinov \& G. Yushin. Science, 334, 75 (2011).

[21] A. Magasinski, B. Zdyrko, I. Kovalenko, B. Hertzberg, R. Burtovvy, C. F. Huebner, T. F. Fuller, I. Luzinov \& G. Yushin. Toward efficient binders for Li-ion battery Si-anodes: polyacrylic acid. Applied Materials and Interfaces, 2(11):3004-3010 (2010).

[22] H. Li, Z. X. Wang, L. Q. Chen \& X. J. Huang. Adv. Mater., 214593 (2009). 
[23] P. H. L. Notten, F. Roozeboom, P. P. L. Regtien, R. A. H. Niessen \& Baggetto. 3-D integrated all-solid-state rechargeable batteries. Adv. MAter., 19:4564-4567 (2007).

[24] G. Ning, B. Haran \& B. N. Popov. Capacity fade study of lithiumion batteries cycled at high discharge rates. J. Power Sources, 117: 160-169 (2003).

[25] N. Nitta, F. Wu, J. T. Lee \& G. Yushin. Li-ion battery materials: present and future. Materials Today, 18(5):252-264 (2015).

[26] J. R. Owen. Rechargeable lithium batteries. Chem. Soc. Rev., 26:259267 (1997).

[27] H. Park, B. Kong \& E. Oh. Effect of high adhesive polyvinyl alcohol binder on the anodes of lithium ion batteries. Electrochemistry Communications, 13(10):1051-1053 (2011).

[28] J. Shim, R. Kostecki, T. Richardson, X. Song \& K. A. Striebel. J. Power Sources, 112:222-230 (2002).

[29] H. Liu, J. M. Foster, A. Gully, S. Krachkovskiy, M. Jiang, Y. Wu, X. Yang, B. Protas, G. Goward \& G. A. Botton. Three-dimensional investigation of cycling-induced microstructural changes in lithium-ion battery cathodes using focused ion beam/scanning electron microscopy. J. Pow. Sou., 306:300-308 (2016).

[30] M. Safari, M. Morcette, A. Teyssot \& C. Delacourt. J. Electrochem. Soc., 156 (2009) A145.

[31] J. Vetter, P. Novak, M. R. Wagner, C. Veit, K. C. Moller, J. O. Besenhard, M. Winter, M. Wohlfahrt-Mehrens, C. Vogler \& A Hammouche. Ageing mechanisms in lithium-ion batteries. Journal of Power Sources, 147:269-281. (2005).

[32] X. Wang, Y. Sone, G. Segami, H. Naito, C. Yamada \& K. Kibe. J. Electrochem. Soc., 154 A14 (2007).

[33] W. Wu, X. Xiao, M. Wang \& X. Huang. A microstructural resolved model for the stress analysis of lithium-ion batteries. J. Electrochem. Soc., 161(5):A803-A813 (2014).

[34] X. Xiao, P. Liu, M. W. Verbrugge, H. Haftbaradaran \& H. Gao. J. Power Sources, 196(3) 1409 (2011). 
[35] X. Xiao, W. Wu \& X. Huang. J. Power Sources, 1957649 (2010). 